\title{
Research of decreasing the influence of coupling effect on High-frequency circular piston transducer
}

\author{
ZHAO Hui ${ }^{1, a}$, WANG Yan ${ }^{2}$, GU Yi-wei ${ }^{1}$, LIU Jia ${ }^{1}$ \\ ${ }^{1}$ Shanghai Marine Electronic Equipment Research Institute, Shanghai 201108, China \\ ${ }^{2}$ underwater sound countermeasure technology key laboratory \\ azhaohui0094@163.com
}

Keywords: high frequency; circular piston; coupling effect

\begin{abstract}
This paper analyzes the impact of coupling effect on the high-frequency circular piston transducer. Focuses on the impact of the direction and the transmission voltage response in the case of constant thickness and mutative diameter. Raised a new method for Circular piezoelectric wafer center opening that decreases the influence of coupling influence, and experimental study was operated. According to the method, Transducer prototype is developed and the influence of coupling influence is little. The resonant peak in the thickness direction is obvious. The new method has a good adaptability, it could be widely extended to the same type of transducer designing.
\end{abstract}

\section{Introduction}

Echo sounder is an important type of underwater acoustic navigation device, it can provide information on the location of the depth of the ship to ensure the safety of navigation. As an important component on the echo sounder, the high-frequency circular piston transducer is mounted on the bottom of ship and is accountable to launch vertically a frequency modulated pulse sonic to underwater.

In practice the high-frequency circular piston transducer is susceptible to coupling effect. Effect of the coupling can be eliminated by the cutting method. But the cutting methods exist shortcomings of more processes, resource-intensive and long-cycle. This paper will propose a method opening hole in the center of the circular ceramic wafers. This method is simple and effective, easily produced, also can effectively avoid the impact of the coupling. The transducer prototype was developed by using the new method, it has only one resonance peak.

\section{Coupling Impact Analysis}

Since the thickness vibration mode frequency is relatively high, so the high-frequency transducers are generally using the thickness vibration mode of the piezoelectric vibrator. Round transducer coupling influence is mainly from the higher-order modes of radial and lateral bending mode, etc. In practice, the high frequency transducer circular transducers can't satisfy the condition that $a$ is much greater than $t$, so that the transducer is often influenced by the coupling Impact.

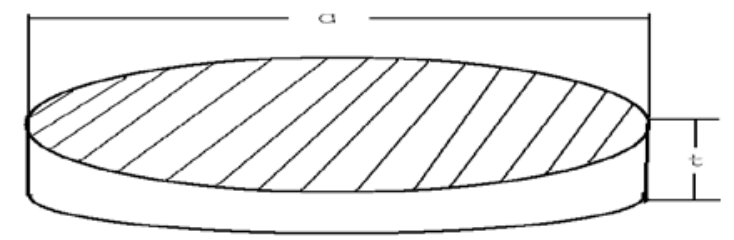

Fig. 1 Circular piezoelectric oscillator schematic

Under ideal conditions, the series resonant frequency of the thickness vibration of PZT4 is

$$
f_{t}=\frac{1.39 v_{t}^{D}}{\pi t}=\frac{2036.3}{t}
$$


Frequency constant, vibration displacement distribution, transmission voltage response of piezoelectric transducers, directivity and other properties were affected by coupling impact. According to the operating frequency of the transducer $f_{t}=218 \mathrm{kHz}$ to calculate, get $t=9.34 \mathrm{~mm}$. Keep $t$ values unchanged, taking a diameter $\mathrm{a}=2 t, 3 t, 5 t, 9 t$ to analysis coupling vibration impact. The paper analysis focused on the voltage response curves and the directivity pattern of transducer changes in dependence of $a$ value.

Figure 2 shows, when $a=2 t$, two obvious resonant peaks are present on the transmit voltage response curve, respectively, $210 \mathrm{kHz}$ and $248 \mathrm{kHz}$, there is a deep valley between the two peaks. When $a=3 \mathrm{t}$, the two resonant peak frequencies are $208 \mathrm{kHz}$ and $240 \mathrm{kHz}$, the valley between the two peaks becomes shallowly.

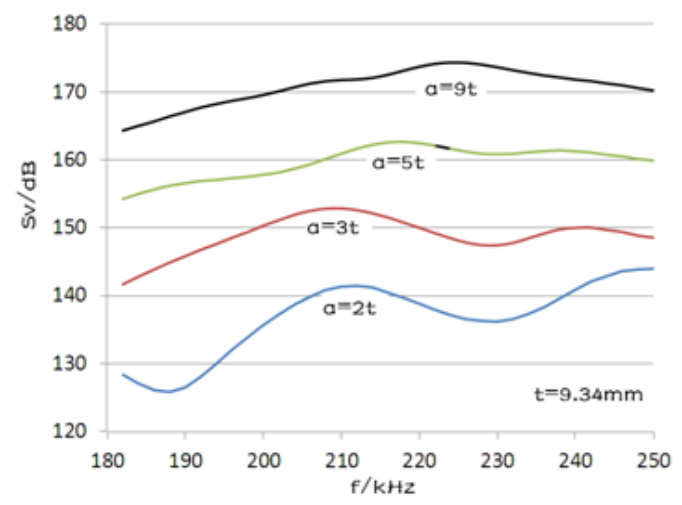

Fig. 2 Transmit voltage response curves at different $a$ values

With the increase of the value $a$, the two resonant peaks frequency gradually move closer, and the valley between two peaks becomes shallowly. When $a=9 t$, the two peaks have been combined to form a resonant peak frequency of $224 \mathrm{kHz}$. This phenomenon shows that when $a=2 t, 3 t, 5 t$, the thickness mode is coupled strongly by the radial vibration high-frequency modes and lateral bending modes. When the $a$ is apposite value, the thickness of the vibration frequency is clear gradually.

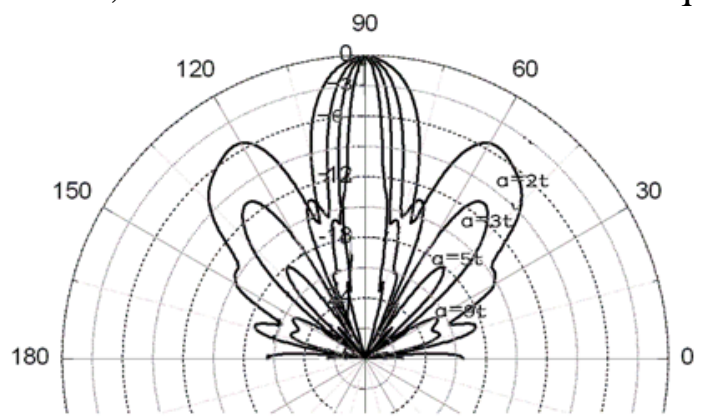

Fig. $3218 \mathrm{kHz}$ directivity pattern at different value $a$

Figure 3 shows, at $218 \mathrm{kHz}, a=2 t,-6 d B$ directivity pattern of an opening angle is $30^{\circ}$, the maximum side lobe level is $-5 d B$. When $a=3 t$, the main beam angle becomes smaller, the maximum side lobe level is $-10.5 d B$, when $a=5 t$, side lobe level is $-18 d B$, a $=9 \mathrm{t}$, side lobe level is $-22.5 d B$. This phenomenon shows that when $a=2 t, 3 t$, the round oscillator is affected by coupling impact. With $a$ increasing, the transducer's directivity pattern gradually is normal, the side lobe level is reducing.Visibly, Coupling Effect in circular piston transducer are: the transmit voltage response curve have multi-resonance peaks, the directivity pattern is abnormal, the side lobe level is higher.

\section{Oscillator center opening effect on coupling}

Keep $t=9.34 \mathrm{~mm}$ and Inner diameter is $5 \mathrm{~mm}$, the outer diameter $a=2 t, 3 t, 4 t, 5 t$, Analysis coupled vibration influence. 


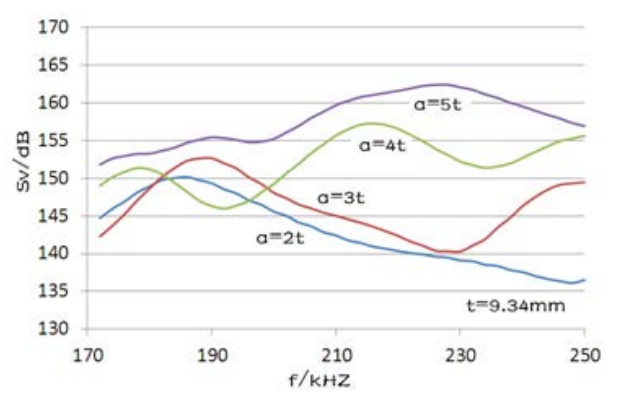

Fig. 4 center opening TVR at different $a \quad$ Fig. 5 center opening $218 \mathrm{kHz}$ directivity pattern When $a=5 t$, the curve has a main peak.Figure 5 shows, when $a=2 t$, the transducer in the $218 \mathrm{kHz}$ directivity pattern $-6 d B$ open angle is $34^{\circ}$, the side lobe level is $-10.1 d B$. when $a=3 t, 4 t$, the maximum side lobe level is $-12 d B, a=5 t$, the side lobe level is $-20 d B$. Compare Figure 3 , the primary beam open-angle has little change, but the low side-lobe level has decreased.

Contrast circular opening hole in the center and not opening two situations: the transducer with center opening easily identify the main resonance peak, and has less impact on the directivity pattern, low side-lobe level.

\section{Experimental study of decoupling}

In practice, the circular piston transducer are limited by mounting dimensions and beam open-angle. The transducer is in the range of coupling, leading to transmit voltage response curve have more resonance peaks. In order to design an operating frequency of $218 \mathrm{kHz}$ circular piston transducer. Uses a circular piezoelectric transducer, the piezoelectric wafer size is $\Phi 60 \times 10 \mathrm{~mm}$, the circular piston transducer 's transmit voltage response data are measured in Figure 6.

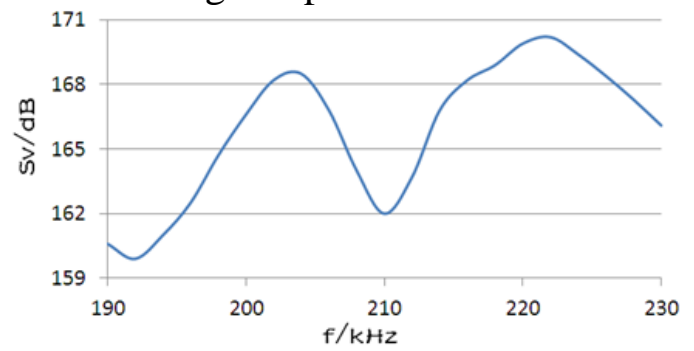

Fig. 6 218kHz transducer's TVR curve

Figure 6 shows that: there are two distinct resonant peaks, and the two peaks are close and the middle of two peaks has a deep valley, the main peak is not easy to discern. To eliminate the effects of coupling action as shown in Fig. 6, according to the principles described earlier, there are two options can be solved. The first is increasing $a$, i.e. the diameter increases. The second is digging a hole at the center of the circular oscillator to reduce the impact of coupling.

After much optimization, the first design of a circular transducer dimension is $\Phi 65 \times 9.8 \mathrm{~mm}$, the test results shown in Figure 7 8.

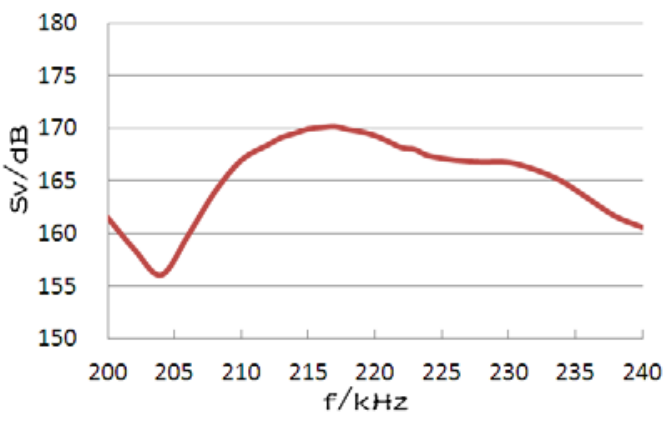

Fig. 7 Ф65 × 9.8 transducer TVR curve

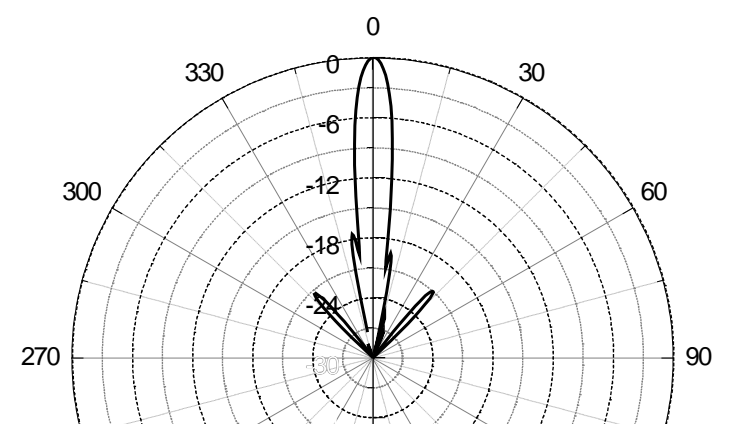

Fig. $8 \Phi 65 \times 9.8218 \mathrm{kHz}$ transducer directivity pattern 
Figure $7-8$ shows that the transducer in the vicinity of $218 \mathrm{kHz}$ has only one main resonance peak and transmit voltage response level is $170 \mathrm{~dB}$.It has less influence by the coupling effect, the beam width of $218 \mathrm{kHz}$ is $6.5^{\circ}$. The side lobe level is $-21 \mathrm{~dB}$.

The second design of the circular transducer dimension is $\Phi 55 \times \Phi 9 \times 9.4 \mathrm{~mm}$, the transducer's test results are shown in Fig. $9 \sim 10$.

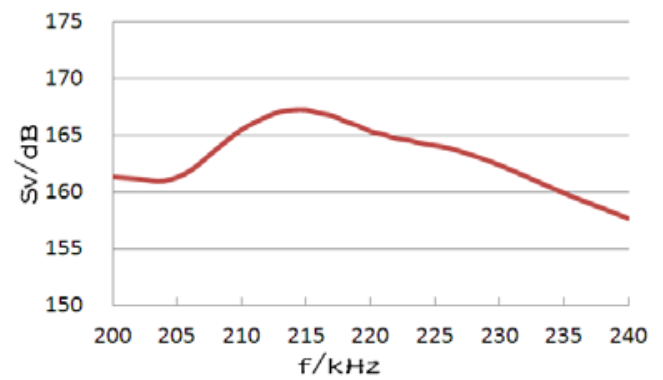

Fig. $9 \Phi 55 \times \Phi 9 \times 9.4$ transducer TVR curve

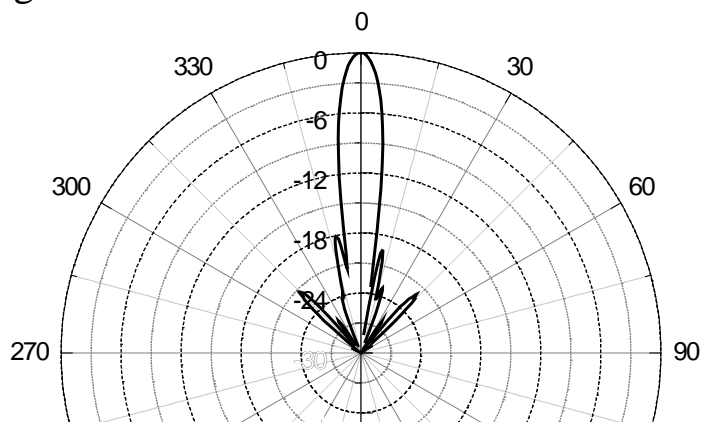

Fig. $10 \Phi 55 \times \Phi 9 \times 9.4218 \mathrm{kHz}$ transducer directivity pattern

Figure $9 \sim 10$ show that, the transducer in the $218 \mathrm{kHz}$ has the main resonance peak significantly and less affected by the coupling effect, the resonance point of the Transmit voltage response is $167 \mathrm{~dB}$, the maximum source level is greater than $205 \mathrm{~dB}$, beam width $218 \mathrm{kHz}$ is $8{ }^{\circ}$, lobe level is $-18 \mathrm{~dB}$.

\section{In conclusion}

This paper analyzes the impact of coupling effect on the high-frequency circular piston transducer, focuses on diameter changes on the transmission voltage response curves and directivity pattern in the thickness of the constant. This paper also analysis the circular oscillator with a hole and without a hole to eliminate coupling effects, and experimental data are described. Two methods are valid to reduce the coupling effects, the paper propose a new method, a new method is simple to reduce coupling influence, it has a good applicability.

\section{References}

[1] LIU Mengan: Underwater sound engineering. Zhe Jiang: Zhe Jiang science technology publication, 2002, p. 377

[2] LIN zhongjian: Composite spherical transducer with high frequency and wide beam width. Applied Acoustics, 2010,29(5):330-335. 